\title{
Translational Machinery in Dendrites of Hippocampal Neurons in Culture
}

\author{
Henri Tiedge ${ }^{1}$ and Jürgen Brosius ${ }^{2}$ \\ ${ }^{1}$ Departments of Pharmacology and Neurology, State University of New York Health Science Center at Brooklyn, \\ Brooklyn, New York 11203, and 2Institute for Experimental Pathology, Center for Molecular Biology of Inflammation, \\ University of Münster, D-48129 Münster, Germany
}

In neurons, several mRNAs are selectively delivered to dendritic domains where they are presumably translated by local protein synthetic machinery. Although electron microscopy has identified polyribosomes in dendrites, in particular in postsynaptic dendritic compartments, the functional composition of the local protein synthetic apparatus and the scope of its translational capacity have not been analyzed. To ascertain the translational competence of dendrites, we have probed hippocampal neurons in primary culture for various integral and associated factors of the translational apparatus. We report here that dendrites of such neurons are equipped with a spectrum of translational machinery components, including ribosomes, tRNAs, initiation and elongation factors, and elements of the cotranslational signal recognition mechanism. These components are differentially and nonuniformly distributed in dendritic arbors. Their dendritic location illustrates the somaindependent potential of dendrites to synthesize selected proteins in local domains.

Key words: dendrite; dendritic RNA; hippocampal neurons; local protein synthesis; ribosomes; translational apparatus; immunocytochemistry; in situ hybridization
An elementary question in cell biology has been how individual cellular components are directed to their local target sites. Classically, proteins synthesized on polyribosomes in the cell body are targeted, co- or post-translationally, to cellular microdomains where they function. As has become increasingly clear in recent years, however, many eukaryotic cell types have developed alternative mechanisms by which specific mRNAs are differentially sorted and targeted to sites of local protein synthesis. Such cell types include, among others, oocytes, fibroblasts, epithelial cells, muscle cells, glial cells, and nerve cells (for review, see Kislauskis and Singer, 1992; Steward and Banker, 1992; Wilhelm and Vale, 1993; Steward, 1994; St Johnston, 1995). In this model, the intracellular distribution of particular proteins would be the consequence of local translation of their cognate mRNAs.

In nerve cells, local translation in dendrites may play a role in the regulation of mosaic dendritic protein pools and consequently in the growth and plasticity of synapses (for review, see Steward and Banker, 1992; Steward, 1994, 1995). Electron microscopy has shown that polyribosomes accumulate beneath synaptic junctions (PalaciosPrü et al., 1981; Steward and Levy, 1982; Bartlett and Banker, 1984b; Steward and Reeves, 1988; Chicurel and Harris, 1992), in particular

Received June 14, 1996; revised Aug. 30, 1996; accepted Sept. 4, 1996.

This work was supported by National Science Foundation Grant IBN-9210149, National Institutes of Health Grant NS34158, and Human Frontier Science Program Organization Grant RG-84/94 B (H.T.). J.B. was supported by the National Institute of Mental Health (MH38819). We are indebted to G. Banker for supplying cultures (prepared by H. Asmussen) and giving invaluable advice concerning experiments and manuscript. G. Banker acknowledges support from National Institutes of Health (NS23094). We thank V. Friedrich for advice on immunocytochemistry, K. Kelley for use of microscopy equipment, and Bio-Rad Laboratories, Molecular Bioscience Group, for access to and assistance with the MRC-1024 Confocal Imaging System. We are grateful to all colleagues who provided antibodies (see Materials and Methods).

Correspondence should be addressed to Henri Tiedge, Department of Pharmacology, State University of New York, Health Science Center at Brooklyn, 450 Clarkson Avenue, Brooklyn, NY 11203.

Copyright (C) 1996 Society for Neuroscience $0270-6474 / 96 / 167171-11 \$ 05.00 / 0$ during periods of developmental and reactive synaptogenesis (Steward, 1983; Steward and Falk, 1986). Additional support for the hypothesis of local translation has come from the identification in dendrites of different types of RNAs, including mRNAs and nonmessenger RNAs. Proteins encoded by dendritic mRNAs belong to diverse classes such as cytoskeletal components (Garner et al., 1988; Bruckenstein et al., 1990; Kleiman et al., 1990; Link et al., 1995; Lyford et al., 1995), kinases (Burgin et al., 1990), and receptors [Furuichi et al., 1993; Miyashiro et al., 1994 (but see Craig et al., 1993); Racca et al., 1996]. Nonmessenger RNAs that have been localized to dendrites include rRNAs (Kleiman et al., 1993, 1994) and the small transcript BC1 RNA (Tiedge et al., 1991; for review, see Brosius and Tiedge, 1995). BC1 RNA has been reported to be complexed with proteins to form a ribonucleoprotein particle of as yet unknown function (Kobayashi et al., 1991; Cheng et al., 1996). Various dendritic RNAs are colocalized in postsynaptic compartments, as was demonstrated by their presence in preparations of dendritic spines and synaptodendrosomes, respectively (Chicurel et al., 1993; Rao and Steward, 1993). De novo synthesis of proteins has been demonstrated directly in preparations of dendrites isolated from hippocampal neurons in culture (Torre and Steward, 1992). Furthermore, it has been shown that glutamatergic synapses upregulate postsynaptic protein synthesis via metabotropic glutamate receptors (Weiler and Greenough, 1993). Taken together, these data suggest that protein synthetic machinery operates in dendritic domains in nerve cells.

Little is known, however, about the molecular composition and translational competence of such machinery. Our overall objective in this study was therefore to ascertain the presence in dendrites of integral and associated components of the protein synthetic machinery. Specifically, we sought to (1) establish the spatial extent to which ribosomes are delivered to the dendritic arborization of a neuron, (2) probe the molecular spectrum of nonribosomal cofactors and associated components (including compo- 

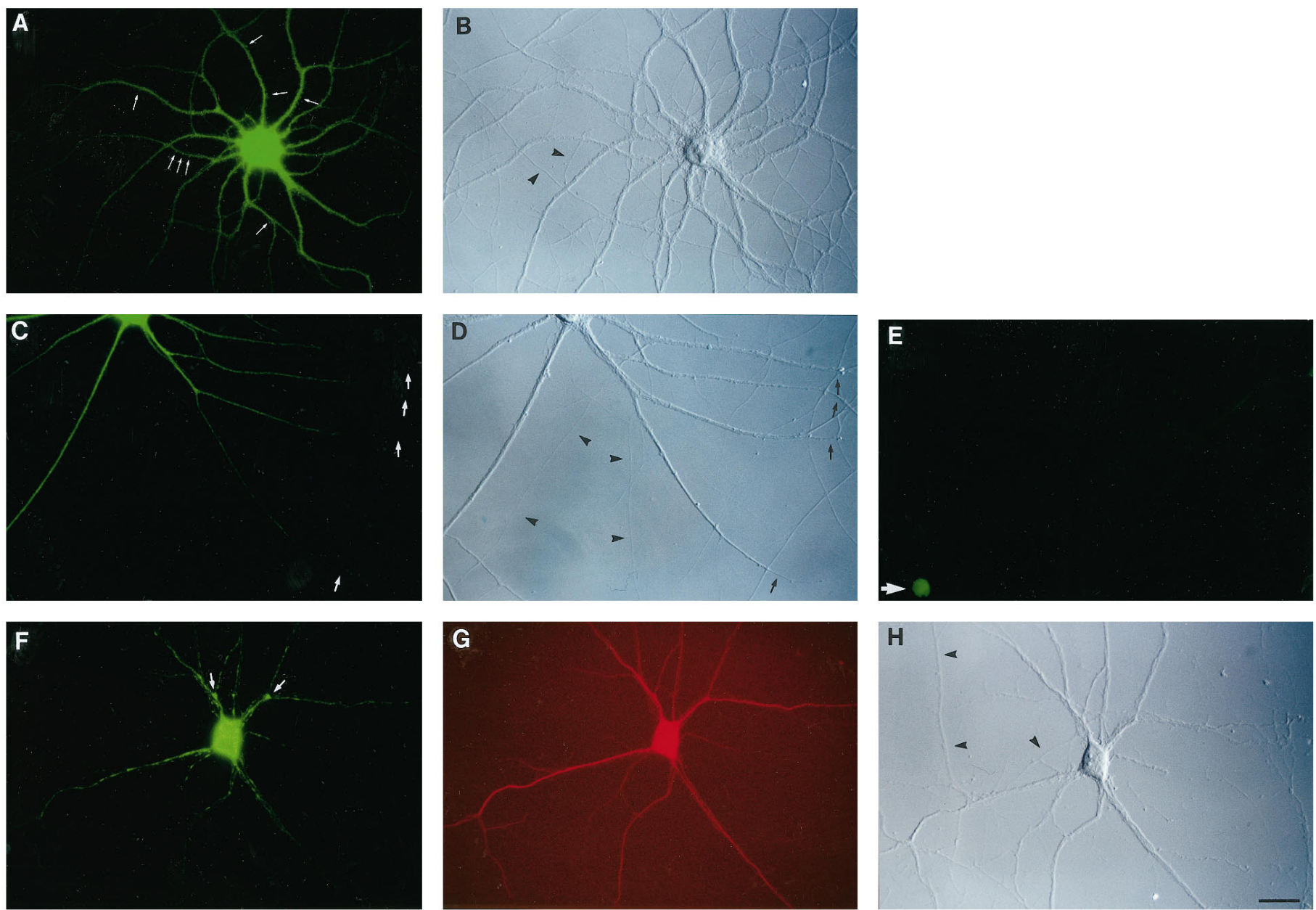

Figure 1. Ribosomes in somatodendritic domains of hippocampal neurons in culture. Neurons were labeled for RPP (green fluorescence; $A$, $C$, E, $F, I$, $L, N)$. Differential interference contrast (DIC) photomicrographs are shown to the right of immunofluorescence photomicrographs $(B, D, H, K, M)$. Arrowheads in DIC fields indicate axons (unlabeled by the anti-RPP antibody). A, Small arrows indicate dendritic segments that bear RPP-labeled spines (barely resolved at this magnification; however, see $N$ ). C, Arrows indicate dendritic segments (RPP-negative) that extend beyond distal branch points and/or intersections. Preincubation of the anti-RPP antibody with purified ribosomes resulted in low-level nuclear (Figure and legend continue.)

nents of the cotranslational protein sorting machinery) that are available to support dendritic protein synthesis, and (3) determine temporal patterns in the dendritic delivery of such components during neuronal development. Here we present data to show that dendrites are supplied with molecular machinery to maintain protein synthetic capacity independent of the soma.

\section{MATERIALS AND METHODS}

Cell culture. Primary cultures of hippocampal neurons were prepared from embryonic rat brains as described (Goslin and Banker, 1991). Briefly, cells were dissociated from prenatal hippocampal tissue (E18) and plated onto poly-L-lysine-treated glass coverslips in minimum essential medium containing $10 \%$ horse serum. After attachment of cells to the substrate, coverslips were transferred to dishes containing monolayer cultures of astroglia so that neurons were facing the layer of glial cells without being in physical contact with them. Cells were then maintained in serum-free medium. Cytosine- $\beta$-D-arabinofuranoside was added $(5 \mu \mathrm{M}$ final concentration) on the fourth day to reduce glial proliferation. At chosen time points, cells were rinsed and fixed in $4 \%$ formaldehyde (freshly made from paraformaldehyde) and $4 \%$ sucrose in PBS (140 mM $\mathrm{NaCl}, 15 \mathrm{~mm}$ phosphate buffer, $\mathrm{pH} 7.3$ ) at $37^{\circ} \mathrm{C}$ for $15 \mathrm{~min}$. Cells were rinsed in PBS and stored in $70 \%$ ethanol at $-20^{\circ} \mathrm{C}$ until further processing. Mature neuronal processes were identified on the basis of their characteristic morphologies and of the differential distribution of MAP2 immunoreactivity, as described by Dotti et al. (1988).

Immunocytochemistry. Fixed cells were rinsed with PBS containing 5
$\mathrm{mM} \mathrm{MgCl}_{2}$ and permeabilized by incubation in "superblock" $(0.5 \mathrm{M}$ Tris/ $\mathrm{HCl}$, pH 7.6, $0.1 \%$ gelatin, $10 \% \mathrm{BSA}, 10 \%$ normal goat serum, and $0.05 \%$ sodium azide) in the presence of $0.25 \%$ Triton X-100 for 10 min at ambient temperature (Godfraind et al., 1989). Cells were then incubated overnight at $4^{\circ} \mathrm{C}$ with a primary antibody or a combination of primary antibodies at appropriate dilutions (see below). After three washes in $0.5 \mathrm{M}$ Tris/ $\mathrm{HCl}, \mathrm{pH} 7.6$ (5 min each, ambient temperature), cells were incubated with species-specific secondary antibodies in $0.5 \mathrm{M}$ Tris/ $\mathrm{HCl}, \mathrm{pH} 7.6,0.1 \%$ gelatin, $1 \% \mathrm{BSA}$, and $0.05 \%$ sodium azide for $2 \mathrm{hr}$ at ambient temperature. We used biotinylated species-specific antibodies (Amersham, Arlington Heights, IL), and for double-labeling with a combination of primary antibodies we used additional species-specific secondary antibodies conjugated with fluorescein or rhodamine derivatives (Jackson, West Grove, PA). Biotinylated secondary antibodies were decorated with streptavidin conjugates (Molecular Probes, Eugene, OR). Coverslips were mounted in $80 \%$ glycerol with $2.5 \%$ 1,4-diazabicyclo[2.2.2]octane (Sigma, St. Louis, MO), and specimens were inspected and photographed with a Nikon Microphot-FX fluorescence microscope. Confocal laser scanning microscopy (CLSM) was performed with a BioRad MRC-1024 Confocal Imaging System.

All primary antibodies that were used in this work have been characterized previously and described in the literature. Primary antibodies were used as follows:

- anti-ribosomal P proteins, human lupus autoantibody, obtained from K. Elkon (Cornell University Medical Center); characterized by Elkon et al. (1985); dilution 1:1000 

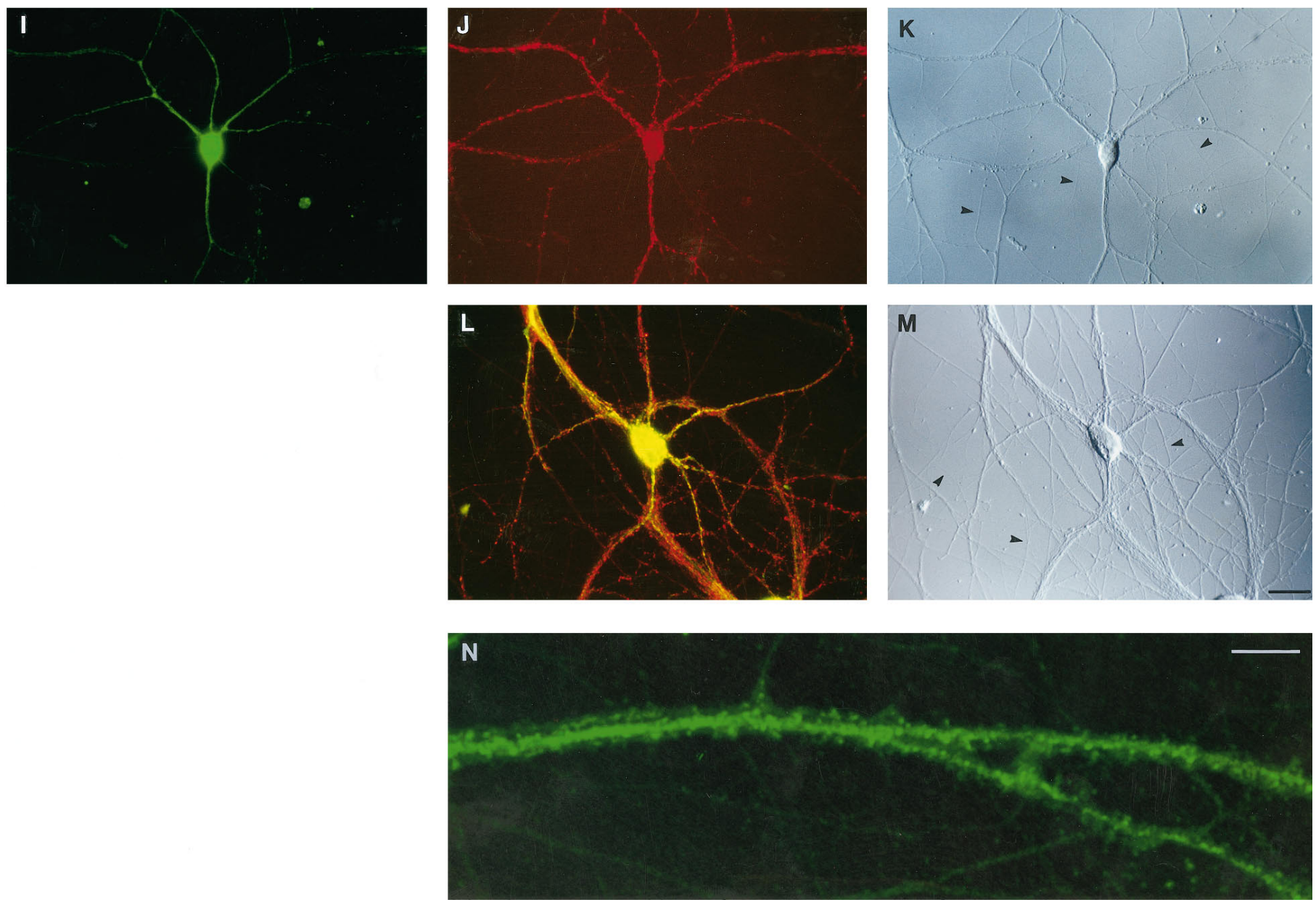

Figure 1 continued.

(Figure 1 continued.) background labeling (E, arrow). F, Arrows indicate clustering of RPP labeling at dendritic branch points. This neuron was double-labeled with an antibody against MAP2 $(G)$. Neuron in I was double-labeled with an antibody against synaptophysin $(J)$. A double exposure of a different neuron labeled with the anti-RPP antibody and the anti-synaptophysin antibody is shown in $L$. CLSM of a dendritic branch labeled with the anti-RPP antibody is shown in $N$. Labeled spine heads appear as knobs protruding from dendritic shafts. Cells in $A-E, N: 28 \mathrm{~d}$ in culture. Cells in $F-M$ : $14 \mathrm{~d}$ in culture. Scale bars: $A-M$, $25 \mu \mathrm{m} ; N, 10 \mu \mathrm{m}$.

-anti-arginyl tRNA synthetase, rabbit polyclonal antibody, affinitypurified, obtained from M. Deutscher (University of Connecticut); characterized by Sivaram et al. (1988); dilution 1:200

-anti-initiation factor $2 \alpha, 2 \beta$, and $2 \gamma$, rabbit polyclonal antibodies, affinity-purified, obtained from U. Bommer (University of London); characterized by Bommer et al. (1988); dilution 1:100

- anti-elongation factor 2, rabbit polyclonal antibody, obtained from A. Nairn (Rockefeller University); characterized by Nairn et al. (1985) and Nairn and Palfrey (1987); dilution 1:1000

- anti-synaptophysin, mouse monoclonal antibody C7.2, obtained from R. Jahn (Yale University); characterized by Jahn et al. (1985); dilution $1: 1000$

- anti-MAP2, mouse monoclonal antibody AP14, obtained from L. Binder (University of Alabama, now Northwestern University Medical School); characterized by Cáceres et al. (1986) and Goslin et al. (1988); dilution 1:200

-anti-TRAP $\alpha$ (anti-SSR $\alpha$ ), rabbit polyclonal antibody, affinity-purified, obtained from T. Rapoport (Max-Delbrück-Center Berlin, now Harvard Medical School); characterized by Görlich et al. (1990); dilution 1:1000 -anti-SRP68, rabbit polyclonal antibody, obtained from P. Walter (University of California, San Francisco); characterized by Walter and Blobel (1983); dilution 1:100

The specificity of all antibodies used in this study has been established in the quoted literature. In addition, the following control experiments were performed to ascertain specificity under our experimental condi- tions. (1) Specificity of primary antibodies was examined by preadsorption with purified antigen in excess, with all subsequent steps in the protocol remaining unchanged (for RPP, ATS, eIF2 $\beta$, TRAP $\alpha$ ). (2) In the case of eEF2 and SRP68, specificity was ascertained by incubation with pre- and nonimmune sera. (3) For all antibodies, background labeling was determined by incubation in the absence of a primary antibody. In background controls for multiple-label experiments, each individual primary antibody was omitted in turn. Secondary antibodies were similarly tested for specificity and lack of cross-species reactivity.

In situ hybridization. Cultured neurons were probed for specific RNAs by in situ hybridization as described earlier (Tiedge, 1991). RNA probes were generated from plasmids that contained the respective sequence of interest cloned into the pBluescript KS (+) vector (Stratagene, La Jolla, CA). Thus, plasmid pRt460 contained the sequence 5'-GTCAGGATGGCCGAGCGGTCTAAGGCGCTGCGTTCAGGTCGCAGTCTC CCCTAGAGGCGTGGGTTCGAATCCCACTCCTGACA-3', which represents full-length unmodified tRNA ${ }^{\text {Leu }}$. Probes specific for 7SL RNA were generated from plasmid pKK451-1, which contained the sequence 5'-CACTAAGTTCGGCATCAATATGGTGACCTC-3'.

This sequence corresponds, in sense orientation, to part of the non-Alu central S region of 7SL RNA (Ullu et al., 1982; Ullu and Tschudi, 1984). Plasmid pKK451-1 was generated by cloning chemically synthesized oligonucleotides into pBluescript KS (+) (Stratagene). High-stringency washes (Tiedge, 1991) were performed at $50^{\circ} \mathrm{C}$ (hybridization to tRNA) and $45^{\circ} \mathrm{C}$ (hybridization to 7SL RNA), respectively. 


\section{RESULTS}

We analyzed the distribution of components of the protein synthetic machinery in hippocampal neurons in primary culture by indirect immunofluorescence and in situ hybridization. We examined the dendritic location of ribosomes, representatives of tRNAs, aminoacyl-tRNA synthetases, and initiation and elongation factors, and of the signal recognition particle (SRP) and translocon-associated protein (TRAP), both markers of the cotranslational signal recognition machinery. The dendritic accumulation of such components was correlated with the development of dendritic arborization and synaptic contacts in cultured hippocampal neurons.

\section{Ribosomes}

To localize ribosomes in hippocampal neurons in primary culture, we used an antibody specific for ribosomal $\mathrm{P}$ proteins (Elkon et al., 1985). In mature neurons, we observed strong ribosomal $\mathrm{P}$ protein (RPP) labeling in cell bodies and in dendrites (Fig. 1). No immunofluorescence signal was detected along axonal processes, indicating that ribosomes are present in such axonal domains at significantly lower levels, if at all, than in somatodendritic domains. This observation is consistent with earlier reports that mature axons contain few, if any, ribosomes (Bartlett and Banker, 1984a,b; Peters et al., 1991). Labeling specificity was ascertained by preadsorption with purified ribosomes. Under such conditions, somatic and dendritic RPP staining was reduced to background levels (Fig. 1E).

In mature dendrites, staining appeared particulate and heterogeneous, at times clustered, for example at dendritic branch points, and at times granular and protruding, thus indicating labeling of dendritic spines. Extreme distal segments were often but not always labeled. When not, staining typically extended to an ultimate branch point or to a distal intersection with another neuronal process, but not beyond (Fig. 1C,D). Clustering at dendritic branch points was often conspicuous in cultures that had not yet reached full maturity (Fig. $1 F-H)$. When such cultures were double-labeled with an antibody against MAP2 (a protein that is restricted to neuronal cell bodies and dendrites; Cáceres et al., 1986; Goslin et al., 1988), the MAP2 signal in dendrites appeared smooth and continuous, whereas axons remained unlabeled (Fig. $1 G)$. RPP immunoreactivity was detectable only in such processes that were also MAP2-positive, a further confirmation that RPP staining was in fact dendritic.

We next analyzed the spatial correlation between RPP labeling and synaptic contacts, using an antibody against synaptophysin (Jahn et al., 1985) to label presynaptic terminals. In cultured hippocampal neurons with mature synapses, this antibody has previously been shown to produce discrete puncta that represent presynaptic specializations (Fletcher et al., 1991, 1994). RPP labeling in such neurons was usually localized to dendritic elements that were targets of presynaptic terminals, as visualized by puncta of synaptophysin staining. RPP-positive dendritic segments were often surrounded and presumably contacted by synaptophysin-labeled presynaptic specializations (Fig. 1I-M). The prominence of RPP labeling in dendritic elements that receive synaptic contacts prompted us to ask whether we could use the RPP antibody to localize ribosomes to dendritic spines at higher resolution, using CLSM. In a superposition of optical sections through part of the dendritic arbor of a mature hippocampal neuron in culture, RPP staining was evident along dendritic shafts and, albeit at a lower level, in numerous dendritic spine heads that were seen protruding from such shafts (Fig. $1 N$ ).
These data illustrate that a subpopulation of dendritic ribosomes is located underneath synaptic structures in dendritic spines.

How are ribosomes delivered to dendrites? Do they accumulate in dendrites as these are being extended, or are they restricted to the soma until a particular signal triggers their targeting to dendrites? We approached these questions by analyzing the dendritic appearance of RPP immunoreactivity in developing hippocampal neurons in culture. Development of these cells proceeds in distinct phases (Dotti et al., 1988). Subsequent to the extension of lamellipodia and minor processes at stages 1 and 2, respectively, axonal outgrowth is initiated at stage 3 (after $\sim 1-2 \mathrm{~d}$ in culture), and dendritic outgrowth is initiated at stage 4 (after $\sim 3-4 \mathrm{~d}$ in culture). Figure 2 shows the spatial progression of RPP immunoreactivity in such cells during a $14 \mathrm{~d}$ period, beginning at day 1 in culture. At this time, cell bodies were strongly labeled. Minor processes showed weaker but significant staining; in the axon, a very weak immunofluorescence signal was observed in the growth cone, in the axonal segment adjacent to it, and in the somaproximal axonal segment. A similar situation was observed at day 2 in culture, with RPP labeling along axonal shafts now at barely detectable levels. After $3 \mathrm{~d}$ in culture, dendritic growth cones had formed; they were clearly RPP-positive (Fig. $2 C$ ). During the next few days in culture, dendritic RPP labeling increased in strength, whereas axonal RPP labeling decreased to levels below detection limits. The developmental pattern indicates that RPP labeling is present in growing neuronal processes, both axonal and dendritic, as these extend growth cones, but is subsequently redistributed in a more polarized fashion such that dendritic labeling continues to increase and axonal labeling vanishes.

At the end of the first week in culture, dendritic RPP staining was robust yet heterogeneous, and axonal labeling was undetectable. During this and subsequent stages of development, RPPpositive clusters were frequently seen to accumulate at dendritic branch points, a pattern that was at times observed to persist in mature cells. Dendritic RPP labeling further increased in intensity and complexity during the second week in culture as dendritic arbors became more elaborate. RPP-positive dendritic spines, rarely seen before $10 \mathrm{~d}$ in culture, were observed in greater numbers after $14 \mathrm{~d}$ in culture (Fig. 2).

\section{tRNA}

Although they are key elements in translation, tRNAs have not been identified in extrasomatic neuronal domains to date. Using a probe against tRNA ${ }^{\text {Leu }}$ for in situ hybridization with hippocampal neurons in primary culture, we observed tRNA labeling in somata and in dendrites but not in axons of mature cells (Fig. 3). Such polarity, however, was not yet obvious in immature cells. After $2 \mathrm{~d}$ in culture, tRNA labeling was apparent in cell bodies, in minor processes, and in the developing axon (Fig. $3 A$ ). In the axon, labeling was concentrated in the growth cone and in segments immediately adjacent to the growth cone; other axonal segments were labeled only lightly. Although in situ hybridization is at best semiquantitative, it is apparent from Figure $3 A$ that a significant fraction of tRNA labeling at this stage was localized to the distal axonal area, including the growth cone. (Note that this may be attributable in part to the larger volume of the axonal growth cone region, as compared with more proximal axonal segments.)

At stage 4, during the second half of the first week in culture, hippocampal neurons extend dendrites and continue to elongate the axon. Dendritic tRNA labeling was detectable as dendrites emerged during this early period of dendritic extension (Fig. $3 B, C)$; it reached substantial levels by the end of the first week in 

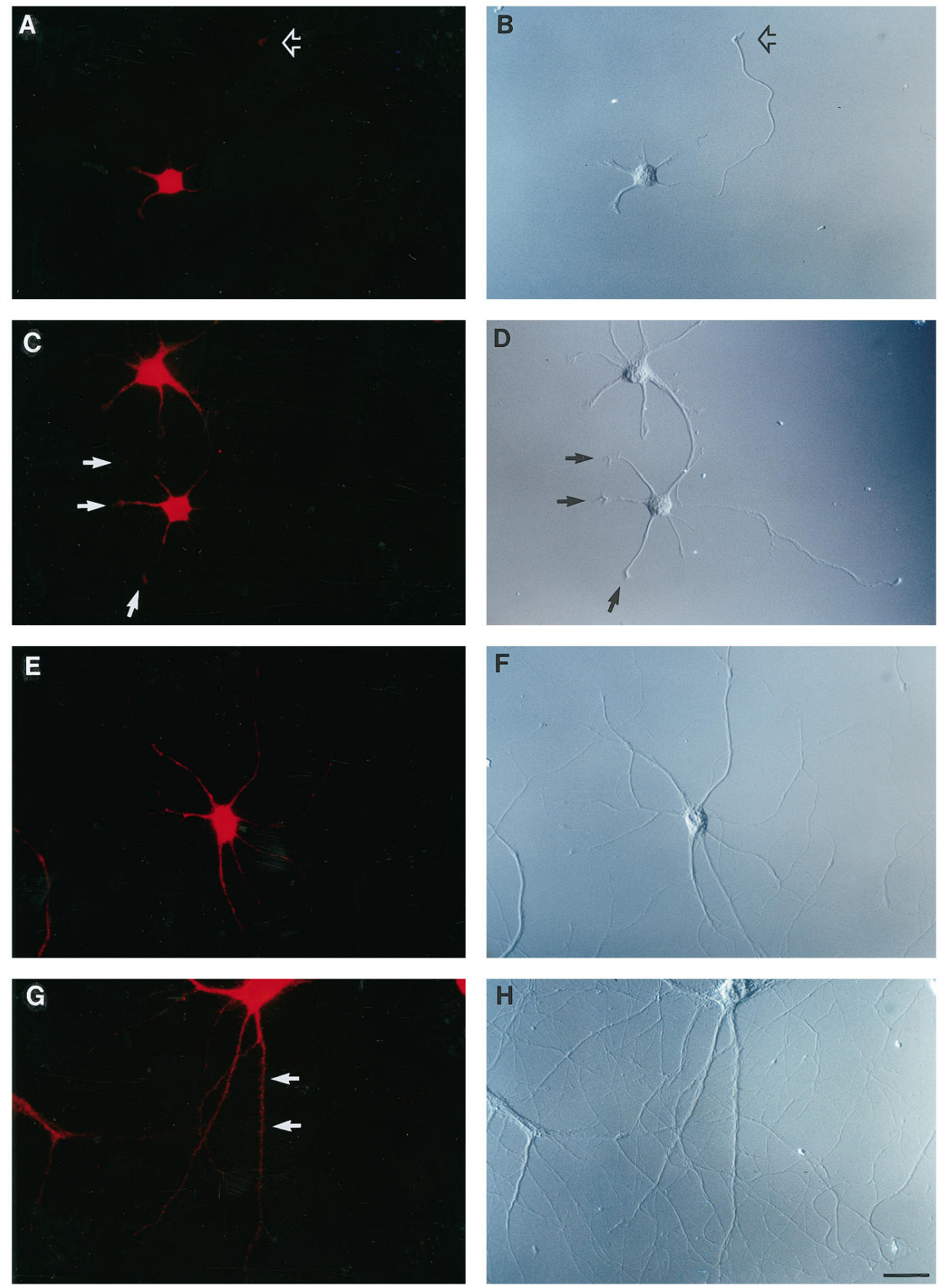

Figure 2. Development of polarized ribosome distribution in cultured hippocampal neurons. Left, Anti-RPP immunofluorescence photomicrographs; right, corresponding DIC photomicrographs. After $1 \mathrm{~d}$ in culture, RPP labeling was prominent in the cell body, to a lesser extent in minor processes, and at low levels in axonal growth cones (open arrowheads in $A, B)$. RPP labeling extended into developing dendrites, including their growth cones (indicated by $a r$ rows for the lower cell in $C, D$ ), after $3 \mathrm{~d}$ in culture. Axonal labeling was barely if at all detectable; it was undetectable at later stages $(E, F, 7 \mathrm{~d}$ in culture; $G, H$, $14 \mathrm{~d}$ in culture). RPP-positive dendritic spines first appeared toward the end of the second week in culture $(G, H)$. A spine-bearing dendritic segment is indicated by arrows in $G$ ). Scale bar, $25 \mu \mathrm{m}$. culture (Fig. 3C). By this time, significant tRNA labeling was no longer detectable in axons. It thus appears that tRNAs, like ribosomes, are initially carried along by the outward-growing axon, but they become effectively excluded from axonal domains as dendrites begin to develop. In more mature neurons, tRNAs assume a polarized distribution, with high levels in somatodendritic domains and levels below detection limits in axonal domains.

\section{Associated components of the translational apparatus}

Amino acid moieties are transferred to tRNAs by aminoacyltRNA synthetases. It has been shown that aminoacyl-tRNAs are not released by the respective synthetase into the surrounding medium but are channeled directly to ribosomes via elongation factor 1 (Negrutskii and Deutscher, 1991; Negrutskii et al., 1994). Therefore, because ribosomes and aminoacyl-tRNA synthetases interact at a short distance, dendritic protein synthesis would require the presence of aminoacyl-tRNA synthetases in dendrites. We used an antibody directed against arginyl-tRNA synthetase (ATS) to examine the distribution of the enzyme in mature hippocampal neurons in culture (Fig. 4A). This antibody labeled cell bodies and dendrites. As with ribosomes and tRNA, axonal processes were unlabeled. ATS labeling in dendrites is heterogeneous and particulate, reminiscent of RPP labeling as shown above.

The presence in dendrites of members of the families of initiation factors and elongation factors, respectively, was analyzed in 

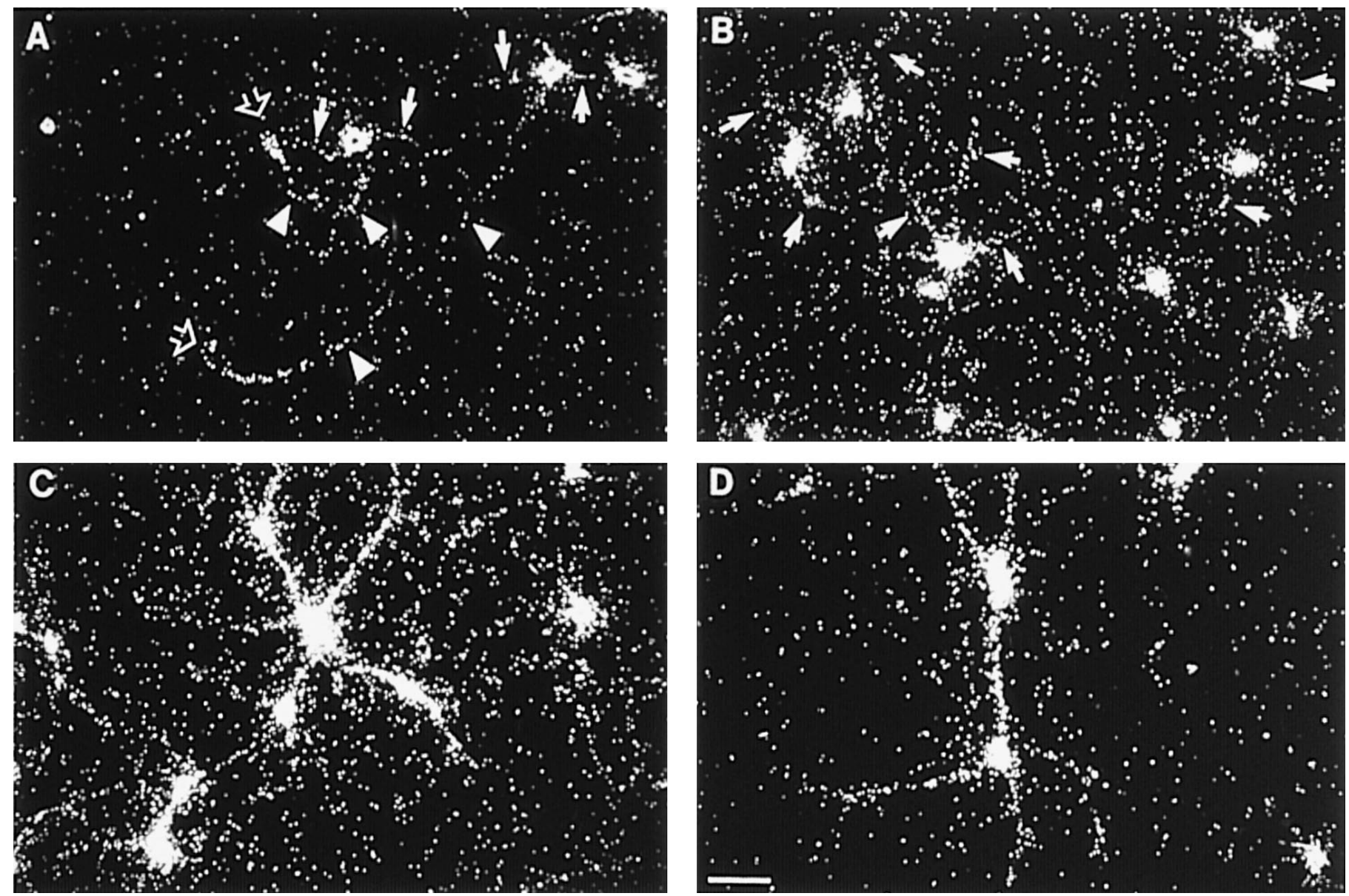

Figure 3. Development of polarized tRNA distribution in cultured hippocampal neurons. In situ hybridization was performed with a probe specific for tRNA $^{\text {Leu }}$. A, At $2 \mathrm{~d}$ in culture, cell bodies were labeled, as were minor processes (arrows), axonal growth cones (open arrowheads), and segments of axonal shafts (arrowheads). B, At $4 \mathrm{~d}$ in culture, labeling was observed in somata and (in a number of cells) in dendrites (arrows), at times extending into growth cones. Dendritic labeling was prominent at $7(C)$ and $14(D) \mathrm{d}$ in culture. At these times, tRNA labeling could be observed in a significant number of dendrites at distances of $150 \mu \mathrm{m}$ or more from the center of the soma. Scale bar, $50 \mu \mathrm{m}$.

the same way. Eukaryotic initiation factor 2 (eIF2) and eukaryotic elongation factor 2 (eEF2) were chosen as examples. eIF2 mediates the binding of the methionyl initiator tRNA to the small ribosomal subunit, whereas eEF2 catalyzes translocation from the A to the $\mathrm{P}$ site on the ribosome. We used antibodies specific for eEF2 and for the $\beta$ subunit of eIF2 (eIF2 $\beta$ ), respectively. In mature hippocampal neurons in culture, both antibodies produced a somatodendritic signal (Fig. 4C,F). Immunofluorescence signals appeared particulate, although less so than RPP or ATS labeling. In double-labeling experiments, eEF2 as well as eIF2 $\beta$ immunoreactivities were localized to dendrites that at the same time were positive for MAP2 (Fig. $4 D$ and data not shown). These data thus suggest that eEF2 and eIF $2 \beta$ are present in somata and in dendrites but not in axonal processes.

\section{Components of the signal recognition mechanism}

Rough endoplasmic reticulum (RER) membranes are the target sites for nascent polypeptide chains destined for membrane integration/translocation. Dendritic synthesis of such proteins would therefore require the presence in dendrites of components of the signal recognition mechanism that mediate membrane targeting and insertion. As markers for this machinery, we chose TRAP [formerly called signal sequence receptor (SSR); see Wiedmann et al., 1987] and the SRP. TRAP is a component of the translocation complex in the RER membrane (Görlich et al., 1990). Here we used an antibody directed against the $\alpha$ subunit to identify this protein in hippocampal neurons in culture. In mature hippocampal neurons, TRAP $\alpha$ labeling was observed in somata and in some but not all dendritic segments; axons were unlabeled (Fig. 5A,B). The immunofluorescence signal was particulate, but less so than with RPP or ATS labeling, and no clear labeling of dendritic spines was observed. TRAP $\alpha$ staining was often restricted to major dendrites or major, proximal segments of dendritic arbors, with considerably less staining in minor or distal branches. Even in major dendrites, labeling was on average significantly lower than in the cell body and was usually restricted to proximal segments. Cells with little or no specific dendritic labeling were also observed occasionally. Specificity of TRAP $\alpha$ labeling was ascertained by preadsorption with the antigen against which the antibody was raised. No specific labeling was observed under those conditions (Fig. 5C).

TRAP $\alpha$ was detected at early stages during development of hippocampal neurons in culture (Fig. $5 D-G$ ). During the first $2 \mathrm{~d}$ in culture, TRAP $\alpha$ staining was predominantly somatic, with little signal detectable in minor processes or in developing axons. By day 4 in culture, a weak dendritic $\operatorname{TRAP} \alpha$ labeling could be observed. Labeling in axonal processes remained very low if at all detectable. No significant labeling was observed in axonal or 

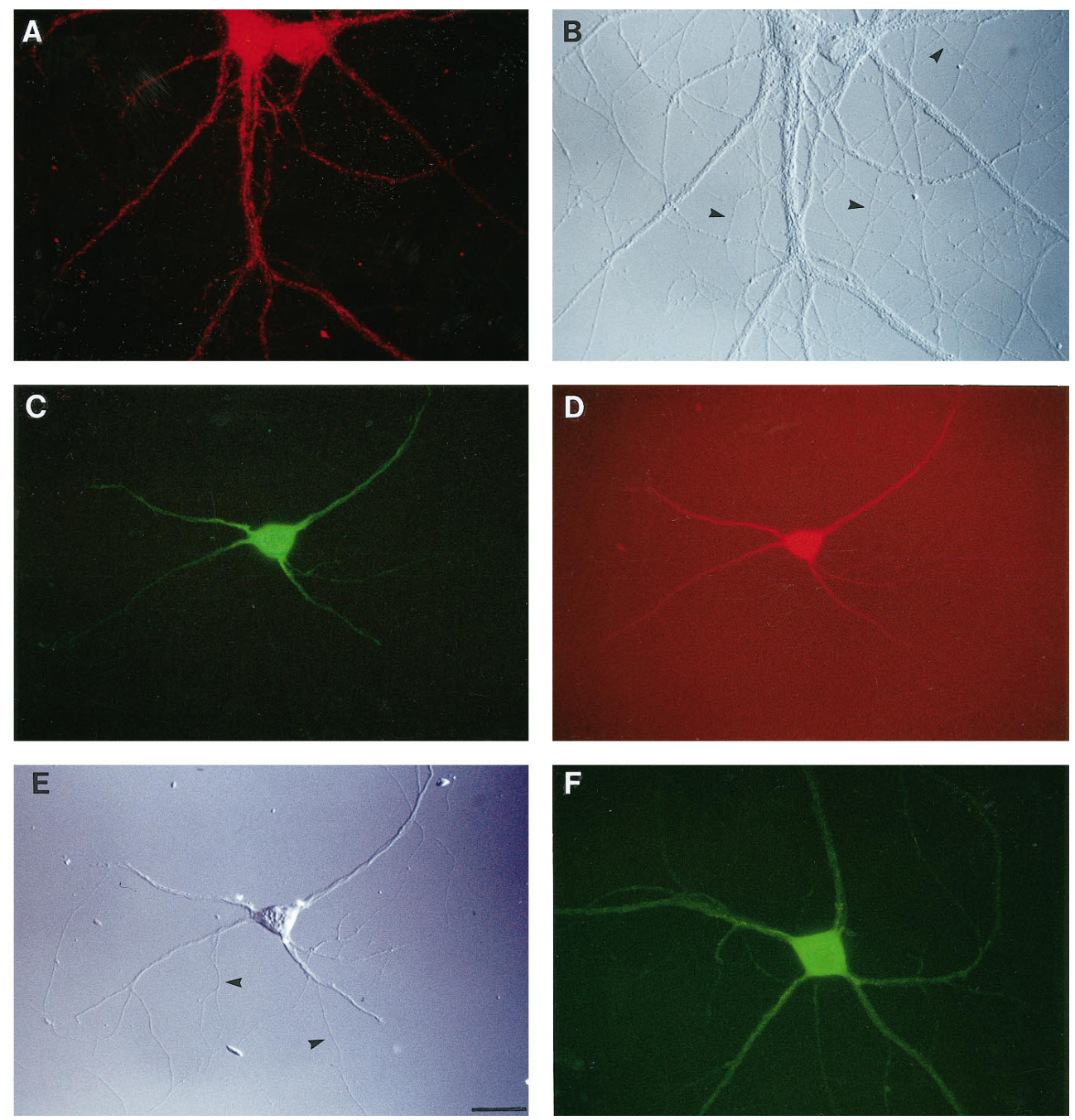

Figure 4. Associated components of the translational apparatus in hippocampal neurons in culture. ATS labeling is shown in $A ; B$ shows the same field in DIC. $C$ shows EF2 labeling; $D$ shows the same cell double-labeled with an anti-MAP2 antibody; $E$ shows the same field in DIC. Labeling for eIF2 $\beta$ is shown in $F$. Cells $28 \mathrm{~d}$ in culture $(A, B), 9 \mathrm{~d}$ in culture $(C-E)$, or $14 \mathrm{~d}$ in culture $(F)$. Arrowheads in DIC photomicrographs indicate unlabeled axons. Scale bar, $25 \mu \mathrm{m}$. dendritic growth cones, a pattern that is in contrast to ribosomal and tRNA labeling at this stage of development. TRAP $\alpha$ labeling was robust in proximal dendrites by the end of the first week in culture and continued to increase in extent and intensity as dendrites matured during the second week in culture (Fig. $5 \mathrm{H}, I$ ). A quantitative comparison of the extent of dendritic $\operatorname{TRAP} \alpha$ labeling during this period (at days 7, 9, and 14 in culture) with the corresponding RPP data confirmed our observation that the latter extended to more distal dendritic domains than the former (Table 1).

The SRP, in a cotranslational process, directs nascent polypeptide chains to RER membranes (for review, see Walter and Johnson, 1994). We analyzed the distribution of the SRP in hippocampal neurons in culture by in situ hybridization with a probe specific for 7SL RNA, the RNA component of the SRP (Walter and Blobel, 1982). Figure 5J,K shows that 7SL RNA was detected at significant levels in mature hippocampal neurons. Labeling was predominantly somatic; significant labeling was apparent in some but not all dendritic segments, whereas axons remained unlabeled. Labeling for 7SL RNA was reminiscent of TRAP labeling in that in mature neurons, labeled dendritic segments were usually proximal rather than distal and of primary rather than higher order; occasionally, labeling was restricted to cell bodies. An antibody directed against SRP protein 68 (Walter and Blobel, 1983) produced similar results
Table 1. Extent of dendritic RPP and TRAP $\alpha$ labeling in hippocampal neurons in culture

Percentage of dendrites labeled at a distance of $100 \mu \mathrm{m}$ from cell body

\begin{tabular}{llll} 
& \multicolumn{4}{l}{$\begin{array}{l}\text { Percentage of dendrites labeled at a distance of } \\
\end{array}$} & $100 \mu \mathrm{m}$ from cell body & \\
\cline { 2 - 4 } & $7 \mathrm{~d}$ in culture & $9 \mathrm{~d}$ in culture & $14 \mathrm{~d}$ in culture \\
\hline RPP labeling & $28(109)$ & $53(113)$ & $79(123)$ \\
TRAP $\alpha$ labeling & $13(183)$ & $35(149)$ & $42(245)$ \\
\hline
\end{tabular}

$\overline{\text { Dendritic RPP or TRAP } \alpha \text { labeling was examined at three time points; labeling was }}$ considered significant if it exceeded background labeling as determined by preadsorption experiments. Listed are the percentages of dendrites that exhibited labeling at a distance of $100 \mu \mathrm{m}$ from the center of the soma. This is followed in parentheses by the respective number of dendrites that were inspected. At $14 \mathrm{~d}$ in culture, the percentage of dendrites labeled for RPP a distance of $200 \mu \mathrm{m}$ from the soma was 52. No TRAP $\alpha$ labeling was detectable at this distance.

(not shown). Both SRP components were detectable in neuronal cell bodies at the earliest stages during development in culture (not shown); however, labeling remained exclusively somatic until neurons had established mature dendritic trees in the course of the second and third week in culture.

\section{DISCUSSION}

Local protein synthesis in dendritic domains is currently being discussed as a novel mechanism for nerve cells to manage den- 

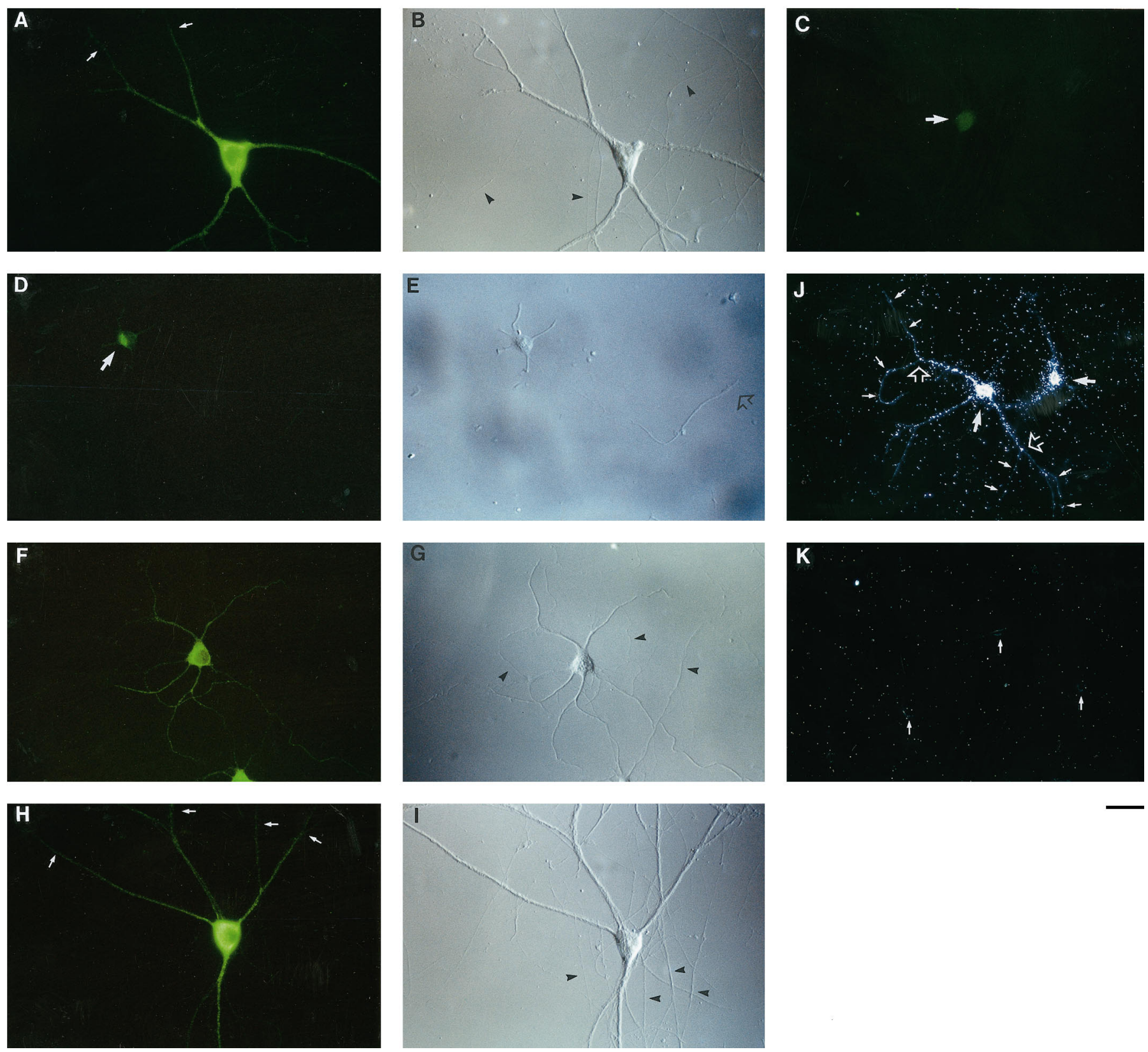

Figure 5. Components of the signal recognition mechanism in cultured hippocampal neurons. Cells labeled with an anti-TRAP $\alpha$ antibody are shown in $A-I$ (anti-TRAP $\alpha$ immunofluorescence photomicrographs and corresponding DIC photomicrographs). After 2 weeks in culture, labeling was observed in soma and proximal dendrites, not however in distal dendrites (arrows in $A$ ) or in axons (arrows in $B$ ). $C$, Preincubation of the anti-TRAP $\alpha$ antibody with the synthetic peptide against which the antibody was raised (Görlich et al., 1990) resulted in low-level nuclear background labeling (arrow). After $1 \mathrm{~d}$ in culture $(D, E)$, TRAP $\alpha$ labeling was restricted to the soma, where it was often localized in a polarized fashion $($ arrow in $D)$. Little or no labeling was observed in minor processes or along axons, including the growth cone (open arrowhead in $E$ ). After $4 \mathrm{~d}$ in culture $(F, G)$, weak dendritic labeling was detectable; axonal labeling remained below detection limits at this and later stages of development $(G, I$, arrowheads). After $14 \mathrm{~d}$ in culture $(H, I)$, labeling was prominent in proximal but not in distal (arrows in $H$ ) dendritic segments. $J$, A probe specific for 7SL RNA labeled somata (arrows) and proximal dendrites, typically up to a distal branch point (open arrowheads). Labeling was often insignificant beyond such branch points (small arrows). $K$, A sense strand control probe failed to produce any significant labeling. Arrows indicate unlabeled neuronal cell bodies. $J, K$, Cells $21 \mathrm{~d}$ in culture. Scale bar: $A-I, 25 \mu \mathrm{m} ; J, K, 50 \mu \mathrm{m}$.

dritic (including postsynaptic) protein repertoires on site, subject to regulation by local physiological stimuli (for review, see Steward, 1995). This notion has gained support from the discovery of an increasing number of dendritic mRNAs (for review, see Steward, 1994, 1995). Because the protein synthetic apparatus necessary for such a mechanism has been poorly defined in dendritic processes, we have in this work addressed the issue of transla- tional machinery in dendrites, using hippocampal neurons in primary culture as a model system. Various different components of the protein synthetic machinery were analyzed, including ribosomes and representatives of tRNAs, aminoacyl-tRNA synthetases, initiation and elongation factors, and components of the cotranslational protein sorting machinery. All components analyzed were identified in dendrites, although distribution patterns 
varied significantly. We conclude that components that are prerequisite for a functionally competent protein synthetic machinery are indeed in place in dendritic domains.

Although the presence of ribosomes in dendrites has been described in early electron micrographs of neurons (for review, see Peters et al., 1991), the significance of this observation was not universally appreciated for a number of years, despite the fact that it was later substantiated through a series of additional electron microscopy studies in which polyribosome clustering in dendrites was shown to be particularly prominent underneath synaptic sites (for review, see Steward and Banker, 1992). In the present study, we have used immunocytochemistry in combination with light microscopy and CLSM to show that ribosomes are present at significant levels throughout the dendritic arborizations of hippocampal neurons in culture. RPP labeling was often seen in distal dendritic tips; at other times, it extended as far as ultimate dendritic branch points or distal intersections with other processes. In mature neurons, labeling was clearly detectable in dendritic spines (albeit at somewhat lower levels than in dendritic shafts), suggesting that a subpopulation of ribosomes is associated with postsynaptic structures.

Associated components of the translational apparatus that were detected in dendrites in our study include representatives of tRNAs, aminoacyl-tRNA synthetases, initiation factors, and elongation factors. Aminoacyl-tRNA synthetases play a key role in chain elongation in that they catalyze the production of aminoacyl-tRNAs and their delivery to the elongating ribosomal complex (for review, see Deutscher, 1984). Our data show that these enzymes, along with elongation factors, are available to participate in translation in dendrites. Of particular interest is the fact that initiation factors could be identified in dendrites. This result suggests that there is no need to preassemble initiation complexes, or in fact entire polyribosomes, in the cell body and transport them to dendritic sites of protein synthesis. Rather, after arrival at their dendritic destinations, mRNAs can be repeatedly translation-initiated in local domains. It is conceivable that initiation and/or elongation in dendrites is regulated by demand. Because both eIF2 and eEF2 have been reported to be mediators of translational regulation in eukaryotes (for review, see Hershey, 1991; Rhoads, 1993), their presence in dendrites may be of particular relevance for the local control of protein synthesis in dendritic domains.

The dendritic location of components of the cotranslational signal recognition mechanism is equally significant. It has been reported that the family of dendritic mRNAs includes members that encode membrane-bound proteins such as the type $1 \mathrm{InsP}_{3}$ receptor (Furuichi et al., 1993), a variety of glutamate receptor subunits (Miyashiro et al., 1994; but compare Craig et al., 1993), and glycine receptor $\alpha$ subunits (Racca et al., 1996). Dendritic translation of such mRNAs would require on-site membrane targeting and insertion of nascent polypeptide chains, and consequently the signal recognition machinery to support this mechanism. The presence in dendritic domains of TRAP and the SRP indicates that products of local translation can indeed be targeted to RER membranes in such domains. TRAP $\alpha$ labeling, in contrast to RPP labeling, frequently failed to extend into minor or distal dendritic branches. Although it can be argued that low staining intensities can result from low-affinity antibody binding, the robust TRAP $\alpha$ signal in cell bodies would suggest that the lower signal intensities in minor or distal dendritic segments is attributable to lower TRAP $\alpha$ protein levels in such domains.

A similar phenomenon, the absence of a labeling signal in minor or distal dendritic segments, was observed with 7SL RNA. Again, it remains unclear at this time whether in such cases the RNA is simply present at levels below in situ hybridization detection limits or whether such dendritic segments do not use SRPbased signal recognition mechanisms. In the latter case, it would be interesting to determine whether alternative signal recognition mechanisms, or none at all, are in operation in such dendritic domains. Although SSR (TRAP) labeling has previously been observed to be confined mostly to neuronal perikarya (KrijnseLocker et al., 1995), our data indicate a more extensive dendritic distribution of this and other components of the signal recognition mechanism. The specificity of TRAP staining in our experiments is confirmed by preadsorption controls and also by the analysis of TRAP labeling during development of hippocampal neurons in culture. Only in mature neurons was TRAP labeling observed in medial/distal dendritic segments; differences in the extent of labeling between this and the previous study (Krijnse-Locker et al., 1995) may thus be attributable in part to different degrees of maturity of the neurons analyzed. Our data are further in line with a most recent report (Racca et al., 1996) that glycine receptor $\alpha$ subunit mRNAs and cisternal components of the Golgi apparatus are localized to dendrites, at significant distances from the soma, in spinal cord neurons in vivo. In earlier work with brain sections, a marker protein of the Golgi complex has been detected, at distances of up to $100 \mu \mathrm{m}$ from the cell body, in large proximal dendrites of neurons in the reticular formation (De Camilli et al., 1986). In more recent work, Golgi markers and protein glycosylation have been observed in major, proximal dendritic segments of hippocampal neurons in culture (Torre and Steward, 1993; Lowenstein et al., 1994; Krijnse-Locker et al., 1995). The combined results thus lead us to conclude that not all translational, cotranslational, and post-translational mechanisms are equally and universally represented in all dendritic subdomains.

It is evident that the subcellular compartmentation of protein synthetic machinery in hippocampal neurons in culture is highly polarized. Generally, negligible levels in axons contrast with high levels in somatodendritic domains, with additional heterogeneity, at least of some components, in dendritic subdomains. Such compartmentation is typical of mature neurons; different distribution patterns of the analyzed components were observed in immature neurons during earlier stages of development. In hippocampal neurons at $1-2 \mathrm{~d}$ in culture, ribosomes and tRNAs were present at high levels in the cell body, and they were clearly detectable in minor processes. Labeling in developing axons was low (in particular for ribosomes) and concentrated in growth cones. At later stages of development, as dendritic processes are being extended, ribosomes and tRNAs accumulate in dendrites and at the same time are reduced along axonal shafts to levels that are below detection limits (which, it should be emphasized, does not indicate complete absence of these components from such domains but rather their presence at levels that are significantly lower than in somata or dendrites; for a discussion of axonal RNAs, see Van Minnen, 1994).

Our data thus suggest that during early stages of neuronal development, subfractions of the cellular ribosome and tRNA populations are passively distributed away from the cell body within growing processes, including the axon. In this scenario, the distribution of these components is later reorganized to dendrites once these begin to acquire their typical characteristics. It remains to be seen what event in the development of a hippocampal neuron triggers the specific delivery of ribosomes and tRNAs to dendrites. It should also be noted at this point that we can 
certainly not rule out the possibility that the dendritic delivery of ribosomes and tRNAs, rather than being a consequence of dendritic differentiation, may be instrumental in triggering such differentiation.

Components of the cotranslational protein sorting machinery seem to be routed to dendrites in a different manner. TRAP and the SRP, although clearly present in somata of immature hippocampal neurons in culture, are barely if at all detectable in minor processes. Little or no specific labeling could be observed for these components at any given point during development in dendritic or axonal growth cones or along axonal shafts. It thus seems that TRAP and the SRP are excluded from nonsomatic domains until they begin to be delivered to dendritic compartments at later stages of development. This observation, and the fact that these two components are not delivered to all dendritic segments in mature neurons, indicates that the way they are sorted to dendrites is different from the sorting of ribosomes and tRNAs. The differential spatiotemporal distribution in dendrites of translational machinery and cotranslational protein targeting machinery thus raises the question, to be investigated in future studies, of whether mRNAs encoding membrane proteins such as receptors are selectively targeted to a specific subset of dendritic polyribosomes. The nonuniformity of mRNA distribution in dendrites, as reported earlier (Miyashiro et al., 1994), would support such a notion.

In conclusion, the results presented here demonstrate that dendrites are equipped with various integral and associated components of the translational apparatus that are required for somaindependent synthesis of proteins, including those that are membrane-associated. Protein synthetic capacity would give dendritic microdomains long-term regulatory competence over local protein repertoires. Although a number of specific mRNAs have been identified in dendrites (for review, see Steward, 1995), it remains to be determined what proteins are actually being synthesized in dendritic microdomains and how their synthesis may be controlled by the physiological status of such compartments.

\section{REFERENCES}

Bartlett WP, Banker GA (1984a) An electron microscopic study of the development of axons and dendrites by hippocampal neurons in culture. I. Cells which develop without intercellular contacts. J Neurosci 4:1944-1953.

Bartlett WP, Banker GA (1984b) An electron microscopic study of the development of axons and dendrites by hippocampal neurons in culture. II. Synaptic relationships. J Neurosci 4:1954-1965.

Bommer U-A, Lutsch G, Behlke J, Stahl J, Nesytova N, Henske A, Bielka H (1988) Shape and location of eukaryotic initiation factor eIF2 on the 40S ribosomal subunit of rat liver. Eur J Biochem 172:653-662.

Brosius J, Tiedge H (1995) Neural BC1 RNA: dendritic localization and transport. In: Localized RNAs (Lipshitz HD, ed), pp 289-300. Austin, TX: RG Landes.

Bruckenstein DA, Lein PJ, Higgins D, Fremeau RT, Jr (1990) Distinct spatial localization of specific mRNAs in cultured sympathetic neurons. Neuron 5:809-819.

Burgin KE, Waxham MN, Rickling S, Westgate SA, Mobley WC, Kelly PT (1990) In situ hybridization histochemistry of $\mathrm{Ca}^{2+} /$ calmodulindependent protein kinase in developing rat brain. $\mathbf{J}$ Neurosci 10:1788-1798.

Cáceres A, Banker GA, Binder L (1986) Immunocytochemical localization of tubulin and microtubule-associated protein 2 during the development of hippocampal neurons in culture. J Neurosci 6:714-722.

Cheng J-G, Tiedge H, Brosius J (1996) Identification of BC1 RNP particles. DNA Cell Biol 15:549-559.

Chicurel ME, Harris KM (1992) Three-dimensional analysis of the structure and composition of CA3 branched dendritic spines and their synaptic relationships with mossy fiber boutons in the rat hippocampus. J Comp Neurol 325:169-182.
Chicurel ME, Terrian DM, Potter H (1993) mRNA at the synapse: analysis of a preparation enriched in hippocampal dendritic spines. J Neurosci 13:4054-4063.

Craig AM, Blackstone CD, Huganir RL, Banker G (1993) The distribution of glutamate receptors in cultured rat hippocampal neurons: postsynaptic clustering of AMPA-selective subunits. Neuron 10:1055-1068.

De Camilli P, Moretti M, Donini SD, Walter U, Lohmann SM (1986) Heterogeneous distribution of the cAMP receptor protein RII in the nervous system: evidence for its intracellular accumulation on microtubules, microtubule-organizing centers, and in the area of the Golgi complex. J Cell Biol 103:189-203.

Deutscher MP (1984) The eukaryotic aminoacyl-tRNA synthetase complex: suggestions for its structure and function. J Cell Biol 99:373-377.

Dotti CG, Sullivan CA, Banker GA (1988) The establishment of polarity by hippocampal neurons in culture. J Neurosci 8:1454-1468.

Elkon KB, Parnassa AP, Foster CL (1985) Lupus autoantibodies target ribosomal P proteins. J Exp Med 162:459-471.

Fletcher TL, Cameron P, De Camilli P, Banker G (1991) The distribution of synapsin I and synaptophysin in hippocampal neurons in culture. J Neurosci 11:1617-1626.

Fletcher TL, De Camilli P, Banker G (1994) Synaptogenesis in hippocampal cultures: evidence indicating that axons and dendrites become competent to form synapses at different stages of neuronal development. J Neurosci 14:6695-6706.

Furuichi T, Simon-Chazottes D, Fujino I, Yamada N, Hasegawa M, Miyawaki A, Yoshikawa S, Guénet J-L, Mikoshiba K (1993) Widespread expression of inositol 1,4,5-trisphosphate receptor type 1 gene (Insp $3 r$ 1) in the mouse central nervous system. Receptors Channels $1: 11-24$.

Garner CC, Tucker RP, Matus A (1988) Selective localization of messenger RNA for cytoskeletal protein MAP2 in dendrites. Nature 336:674-677.

Godfraind C, Friedrich Jr VL, Dubois-Dalcq M (1989) In vivo analysis of glial cell phenotypes during a demyelinating disease in mice. J Cell Biol 109:2405-2416.

Görlich D, Prehn S, Hartmann E, Herz J, Otto A, Kraft R, Wiedmann M, Knespel S, Dobberstein B, Rapoport TA (1990) The signal sequence receptor has a second subunit and is part of a translocational complex in the endoplasmic reticulum as probed by bifunctional reagents. J Cell Biol 111:2283-2294.

Goslin K, Banker G (1991) Rat hippocampal neurons in low density cultures. In: Culturing nerve cells (Banker G, Goslin K, eds), pp 251-282. Cambridge, MA: MIT.

Goslin K, Schreyer DJ, Skene JHP, Banker G (1988) Development of neuronal polarity: GAP-43 distinguishes axonal from dendritic growth cones. Nature 336:672-674.

Hershey JWB (1991) Translational control in mammalian cells. Annu Rev Biochem 60:717-755.

Jahn R, Schiebler W, Ouimet C, Greengard P (1985) A 38,000-dalton membrane protein (p38) present in synaptic vesicles. Proc Natl Acad Sci USA 82:4137-4141.

Kislauskis EH, Singer RH (1992) Determinants of mRNA localization. Curr Opin Cell Biol 4:975-978.

Kleiman R, Banker G, Steward O (1990) Differential subcellular localization of particular mRNAs in hippocampal neurons in culture. Neuron 5:821-830.

Kleiman R, Banker G, Steward O (1993) Subcellular distribution of rRNA and poly (A) RNA in hippocampal neurons in culture. Mol Brain Res 20:305-312.

Kleiman R, Banker G, Steward O (1994) Development of subcellular mRNA compartmentation in hippocampal neurons in culture. J Neurosci 14:1130-1140.

Kobayashi S, Goto S, Anzai K (1991) Brain-specific small RNA transcript of the identifier sequences is present as a $10 \mathrm{~S}$ ribonucleoprotein particle. J Biol Chem 266:4726-4730.

Krijnse-Locker J, Parton RP, Fuller SD, Griffiths G, Dotti CG (1995) The organization of the endoplasmic reticulum and the intermediate compartment in cultured rat hippocampal neurons. Mol Biol Cell 6:1315-1332.

Link W, Konietzko U, Kauselmann G, Krug M, Schwanke B, Frey U, Kuhl D (1995) Somatodendritic expression of an immediate early gene is regulated by synaptic activity. Proc Natl Acad Sci USA 92:5734-5738. Lowenstein PR, Morrison EE, Bain D, Shering AF, Banting G, Douglas P, Castro MG (1994) Polarized distribution of the Trans-Golgi Network 
marker TGN38 during the in vitro development of neocortical neurons: effects of nocodazole and brefeldin A. Eur J Neurosci 6:1453-1465.

Lyford GL, Yamagata K, Kaufmann WE, Barnes CA, Sanders LK, Copeland NG, Gilbert DJ, Jenkins NA, Lanahan AA, Worley PF (1995) Arc, a growth factor and activity-regulated gene, encodes a novel cytoskeleton-associated protein that is enriched in neuronal dendrites. Neuron 14:433-445.

Miyashiro K, Dichter M, Eberwine J (1994) On the nature and differential distribution of mRNAs in hippocampal neurites: implications for neuronal functioning. Proc Natl Acad Sci USA 91:10800-10804.

Nairn AC, Palfrey HC (1987) Identification of the major $M_{r} 100,000$ substrate for calmodulin-dependent protein kinase III in mammalian cells as elongation factor-2. J Biol Chem 262:17299-17303.

Nairn AC, Bhagat B, Palfrey HC (1985) Identification of calmodulindependent protein kinase III and its major $M_{r} 100,000$ substrate in mammalian tissues. Proc Natl Acad Sci USA 82:7939-7943.

Negrutskii BS, Deutscher MP (1991) Channeling of aminoacyl-tRNA for protein synthesis in vivo. Proc Natl Acad Sci USA 88:4991-4995.

Negrutskii BS, Stapulionis R, Deutscher MP (1994) Supramolecular organization of the mammalian translation system. Proc Natl Acad Sci USA 91:964-968.

Palacios-Prü EL, Palacios L, Mendoza RV (1981) Synaptogenetic mechanisms during chick cerebellar cortex development. Submicrosc Cytol 13:145-167.

Peters A, Palay SL, Webster H deF (1991) The fine structure of the nervous system. Neurons and their supporting cells, 3rd edition. New York: Oxford UP.

Racca C, Gardio A, Triller A (1996) Dendritic localization of glycine receptor $\alpha$ subunit mRNAs. Abstract presented at the 16th Blankenese Conference "Intracellular trafficking of protein and RNA," Hamburg, Germany.

Rao A, Steward O (1993) Evaluation of RNAs present in synaptodendrosomes: dendritic, glial, and neuronal cell body contribution. J Neurochem 61:835-844.

Rhoads RE (1993) Regulation of eukaryotic protein synthesis by initiation factors. J Biol Chem 268:3017-3020.

Sivaram P, Vellekamp G, Deutscher MP (1988) A role for lipids in the functional and structural properties of the rat liver aminoacyl-tRNA synthetase complex. J Biol Chem 263:18891-18896.

St Johnston D (1995) The intracellular localization of messenger RNA. Cell 81:161-170.

Steward O (1983) Alterations in polyribosomes associated with dendritic spines during the reinnervation of the dentate gyrus of the adult rat. J Neurosci 3:177-188.

Steward O (1994) Dendrites as compartments for macromolecular synthesis. Proc Natl Acad Sci USA 91:10766-10768.

Steward O (1995) Targeting of mRNAs to subsynaptic microdomains in dendrites. Curr Opin Cell Biol 5:55-61.

Steward O, Banker GA (1992) Getting the message from the gene to the synapse: sorting and intracellular transport of RNA in neurons. Trends Neurosci 15:180-186.

Steward O, Falk PM (1986) Protein synthetic machinery at postsynaptic sites during synaptogenesis: a quantitative study of the association between polyribosomes and developing synapses. J Neurosci 6:412-423.

Steward O, Levy WB (1982) Preferential localization of polyribosomes under the base of dendritic spines in granule cells of the dentate gyrus. J Neurosci 2:284-291.

Steward O, Reeves TM (1988) Protein-synthetic machinery beneath postsynaptic sites on CNS neurons: association between polyribosomes and other organelles at the synaptic site. J Neurosci 8:176-184.

Tiedge H (1991) The use of UV light as a cross-linking agent for cells and tissue sections in in situ hybridization. DNA Cell Biol 10:143-147.

Tiedge H, Fremeau Jr RT, Weinstock PH, Arancio O, Brosius J (1991) Dendritic location of neural BC1 RNA. Proc Natl Acad Sci USA 88:2093-2097.

Torre ER, Steward O (1992) Demonstration of local protein synthesis within dendrites using a new cell culture system which permits the isolation of living axons and dendrites from their cell bodies. J Neurosci 12:762-772.

Torre ER, Steward O (1993) Posttranslational modifications of proteins within dendrites: evaluation of sugar incorporation in isolated dendrites from neurons in culture. Soc Neurosci Abstr 19:63.

Ullu E, Tschudi C (1984) Alu sequences are processed 7SL RNA genes. Nature 312:171-172.

Ullu E, Murphy S, Melli M (1982) Human 7SL RNA consists of a 140 nucleotide middle-repetitive sequence inserted in an Alu sequence. Cell 29:195-202.

Van Minnen J (1994) RNA in the axonal domain: a new dimension in neuronal functioning? Histochem J 26:377-391.

Walter P, Blobel G (1982) Signal recognition particle contains a 7S RNA essential for protein translocation across the endoplasmic reticulum. Nature 299:691-698.

Walter P, Blobel G (1983) Subcellular distribution of signal recognition particle and 7SL-RNA determined with polypeptide-specific antibodies and complementary DNA probe. J Cell Biol 97:1693-1699.

Walter P, Johnson AE (1994) Signal sequence recognition and protein targeting to the endoplasmic reticulum membrane. Annu Rev Cell Biol 10:87-119.

Weiler IJ, Greenough WT (1993) Metabotropic glutamate receptors trigger postsynaptic protein synthesis. Proc Natl Acad Sci USA 90:7168-7171.

Wiedmann M, Kurzchalia TV, Hartmann E, Rapoport TA (1987) A signal sequence receptor in the endoplasmic reticulum membrane. Nature 328:830-833.

Wilhelm JE, Vale RD (1993) RNA on the move: the mRNA localization pathway. J Cell Biol 123:269-274. 